\title{
Minimum Error Entropy Kalman Filter
}

\author{
Badong Chen ${ }^{\text {a }}$, Lujuan Dang ${ }^{\mathrm{a}}$, Yuantao Gu ${ }^{\mathrm{b}}$, Nanning Zheng ${ }^{\mathrm{a}}$, José C. Príncipe ${ }^{\mathrm{a}, \mathrm{c}}$ \\ ${ }^{\mathrm{a}}$ Institute of Artificial Intelligence and Robotics, Xi'an Jiaotong University, Xi'an, 710049, China \\ ${ }^{\mathrm{b}}$ Beijing National Research Center for Information Science and Technology (BNRist) and Department of Electronic \\ Engineering, Tsinghua University, Beijing, 100084, China \\ ${ }^{\mathrm{c}}$ Department of Electrical and Computer Engineering, University of Florida, Gainesville, FL, 32611, USA
}

\begin{abstract}
To date most linear and nonlinear Kalman filters (KFs) have been developed under the Gaussian assumption and the wellknown minimum mean square error (MMSE) criterion. In order to improve the robustness with respect to impulsive (or heavy-tailed) non-Gaussian noises, the maximum correntropy criterion (MCC) has recently been used to replace the MMSE criterion in developing several robust Kalman-type filters. To deal with more complicated non-Gaussian noises such as noises from multimodal distributions, in the present paper we develop a new Kalman-type filter, called minimum error entropy Kalman filter (MEE-KF), by using the minimum error entropy (MEE) criterion instead of the MMSE or MCC. Similar to the MCC based KFs, the proposed filter is also an online algorithm with recursive process, in which the propagation equations are used to give prior estimates of the state and covariance matrix, and a fixed-point algorithm is used to update the posterior estimates. In addition, the minimum error entropy extended Kalman filter (MEE-EKF) is also developed for performance improvement in the nonlinear situations. The high accuracy and strong robustness of MEE-KF and MEE-EKF are confirmed by experimental results.
\end{abstract}

Key words: Kalman filtering, Minimum Error Entropy (MEE), robust estimation, non-Gaussian noises.

\section{Introduction}

Kalman filtering is a powerful technology for estimating the states of a dynamic system, which finds applications in many areas including navigation, guidance, data integration, pattern recognition, tracking and control systems $[1-5]$. The original Kalman filter (KF) was derived for a linear state space model with Gaussian assumption $[2,6]$. To cope with nonlinear estimation problems, a variety of nonlinear extensions of the original Kalman filter have been proposed in the literature, including extended Kalman filter (EKF) $[7,8]$, unscented Kalman filter (UKF) [9], cubature Kalman filter (CKF) [10] and many others. However, most of these Kalman filters are developed based on the popular minimum mean square

\footnotetext{
* This paper was not presented at any IFAC meeting. Corresponding author Badong Chen. Tel. 86-29-82668802 ext.8009. Fax. 86-29-82668672.

Email addresses: chenbd@mail.xjtu.edu.cn (Badong Chen), danglj@stu.xjtu.edu.cn (Lujuan Dang), gyt@tsinghua.edu.cn (Yuantao Gu), nnzheng@mail.xjtu.edu.cn (Nanning Zheng), principe@cnel.ufl.edu (José C. Príncipe).
}

error (MMSE) criterion and will face performance degradation in case of complicated noises, since in general MMSE is not a good choice for the estimation in nonGaussian noises.

In recent years, to solve the performance degradation problem in heavy-tailed (or impulsive) non-Gaussian noises, some robust Kalman filters have been developed by using certain non-MMSE criterion as the optimality criterion [11, 12]. Particularly, the maximum correntropy criterion (MCC) $[13,14]$ in information theoretic learning (ITL) $[11,12]$ has been successfully applied in Kalman filtering to improve the robustness against impulsive noises. Typical examples include the maximum correntropy based Kalman filters [15-24], maximum correntropy based extended Kalman filters [25-27], maximum correntropy based unscented Kalman filters [28-30], maximum correntropy based square-root cubature Kalman filters [31,32] and so on. Since correntropy is a local similarity measure and insensitive to large errors, these MCC based filters are little influenced by large outliers $[13,33]$.

The MCC is a nice choice for dealing with heavy-tailed 
non-Gaussian noises, but its performance may not be good when facing more complicated non-Gaussian noises, such as noises from multimodal distributions. The minimum error entropy (MEE) criterion $[34,35]$ is another important learning criterion in ITL, which has been successfully applied in robust regression, classification, system identification and adaptive filtering [34-38]. Numerous experimental results show that MEE can outperform MCC in many situations although its computational complexity is a little higher $[39,40]$. In addition, the superior performance and robustness of MEE have been proved in [41]. The goal of this work is to develop a new Kalman-type filter, called minimum error entropy Kalman filter (MEE-KF), by using the MEE as the optimality criterion. The proposed filter uses the propagation equations to obtain the prior estimates of the state and covariance matrix, and a fixed-point algorithm to update the posterior estimates and covariance matrix, recursively and online. To further improve the performance in the nonlinear situations, the MEE criterion is also incorporated into EKF, resulting in minimum error entropy extended Kalman filter (MEE-EKF).

The rest of the paper is organized as follows. In section II, we briefly review the KF algorithm and MEE criterion. In section III, we develop the MEE-KF algorithm. Sections IV and V provide the computational complexity and convergence analysis, respectively. In section VI, the MEE-EKF is developed. The experimental results are presented in section VII and finally, the conclusion is given in section VIII.

\section{Background}

\subsection{Kalman Filter}

Consider a linear dynamic system with unknown state vector $\mathbf{x}(k) \in \mathbb{R}^{n \times 1}$ and available measurement vector $\mathbf{y}(k) \in \mathbb{R}^{m \times 1}$. To estimate the state $\mathbf{x}(k)$, Kalman filter (KF) assumes a state space model described by the following state and measurement equations:

$$
\begin{aligned}
& \mathbf{x}(k)=\mathbf{F x}(k-1)+\mathbf{q}(k-1) \\
& \mathbf{y}(k)=\mathbf{H} \mathbf{x}(k)+\mathbf{r}(k),
\end{aligned}
$$

where $\mathbf{F} \in \mathbb{R}^{n \times n}$ and $\mathbf{H} \in \mathbb{R}^{m \times n}$ are the state-transition matrix and measurement matrix, respectively. Here, the process noise $\mathbf{q}(k-1) \in \mathbb{R}^{n \times 1}$ and measurement noise $\mathbf{r}(k) \in \mathbb{R}^{m \times 1}$ are mutually independent, and satisfy

$$
\begin{aligned}
& E[\mathbf{q}(k-1)]=0 ; E[\mathbf{r}(k)]=0 \\
& E\left[\mathbf{q}(k-1) \mathbf{q}^{\mathrm{T}}(k-1)\right]=\mathbf{Q}(k-1) \\
& E\left[\mathbf{r}(k) \mathbf{r}^{\mathrm{T}}(k)\right]=\mathbf{R}(k),
\end{aligned}
$$

where $\mathbf{Q}(k-1)$ and $\mathbf{R}(k)$ are the covariance matrices of $\mathbf{q}(k-1)$ and $\mathbf{r}(k)$, respectively. In general, the $\mathrm{KF}$ includes two steps:
(1) Predict: The a-priori estimate $\hat{\mathbf{x}}(k \mid k-1)$ and the corresponding error covariance matrix $\mathbf{P}(k \mid k-1)$ are calculated by

$$
\begin{aligned}
& \hat{\mathbf{x}}(k \mid k-1)=\mathbf{F} \hat{\mathbf{x}}(k-1) \\
& \mathbf{P}(k \mid k-1)=\mathbf{F P}(k-1) \mathbf{F}^{\mathrm{T}}+\mathbf{Q}(k-1) .
\end{aligned}
$$

(2) Update: The a-posteriori estimate $\hat{\mathbf{x}}(k)$ and the corresponding error covariance matrix $\mathbf{P}(k)$ are obtained by

$$
\begin{aligned}
\hat{\mathbf{x}}(k) & =\hat{\mathbf{x}}(k \mid k-1)+\mathbf{K}(k)[\mathbf{y}(k)-\mathbf{H} \hat{\mathbf{x}}(k \mid k-1)] \\
\mathbf{K}(k)= & \mathbf{P}(k \mid k-1) \mathbf{H}^{\mathrm{T}}\left[\mathbf{H P}(k \mid k-1) \mathbf{H}^{\mathrm{T}}+\mathbf{R}(k)\right]^{-1} \\
\mathbf{P}(k)= & {[\mathbf{I}-\mathbf{K}(k) \mathbf{H}] \mathbf{P}(k \mid k-1)[\mathbf{I}-\mathbf{K}(k) \mathbf{H}]^{\mathrm{T}} } \\
& +\mathbf{K}(k) \mathbf{R}(k) \mathbf{K}^{\mathrm{T}}(k),
\end{aligned}
$$

where $\mathbf{K}(k)$ is the Kalman filter gain.

\subsection{Minimum Error Entropy Criterion}

Different from the MMSE [34] and MCC [35], the MEE aims to minimize the information contained in the error. In MEE, the error information $e$ can be measured by the Renyi's entropy:

$$
H_{\alpha}(e)=\frac{1}{1-\alpha} \log V_{\alpha}(e),
$$

where $\alpha(\alpha \neq 1, \alpha>0)$ is the order of Renyi's entropy, and $V_{\alpha}(e)$ denotes the information potential defined by

$$
V_{\alpha}(e)=\int p^{\alpha}(x) d x=E\left[p^{\alpha-1}(e)\right]
$$

where $p($.$) is the probability density function (PDF) of$ error $e$ and $E[\cdot]$ denotes the expectation operator. In practical applications, the $\operatorname{PDF} p(x)$ can be estimated by Parzen's window approach [11]:

$$
\hat{p}(x)=\frac{1}{N} \sum_{i=1}^{N} G_{\sigma}\left(x-e_{i}\right),
$$

where $G_{\sigma}(x)=\frac{1}{\sqrt{2 \pi} \sigma} \exp \left(\frac{-(x)^{2}}{2 \sigma^{2}}\right)$ denotes the Gaussian kernel with kernel size $\sigma ;\left\{e_{i}\right\}_{i=1}^{N}$ are $N$ error samples. Combining (12) and (13), one can obtain an estimate of the second order $(\alpha=2)$ information potential $V_{2}(e)$ :

$$
\hat{V}_{2}(e)=\frac{1}{N} \sum_{i=1}^{N} \hat{p}\left(e_{i}\right)=\frac{1}{N^{2}} \sum_{i=1}^{N} \sum_{j=1}^{N} G_{\sigma}\left(e_{i}-e_{j}\right)
$$

Since the negative logarithmic function $-\log$ is monotonically decreasing, minimizing the error entropy $H_{2}(e)$ means maximizing the information potential $\hat{V}_{2}(e)$. 


\section{Minimum Error Entropy Kalman Filter}

\subsection{Augmented Model}

First, we denote the state prediction error as

$$
\boldsymbol{\epsilon}(k \mid k-1)=\mathbf{x}(k)-\hat{\mathbf{x}}(k \mid k-1) .
$$

Combining the above state prediction error with the measurement equation (2), one can obtain an augmented model

$$
\left[\begin{array}{l}
\hat{\mathbf{x}}(k \mid k-1) \\
\mathbf{y}(k)
\end{array}\right]=\left[\begin{array}{l}
\mathbf{I}_{n} \\
\mathbf{H}
\end{array}\right] \mathbf{x}(k)+\mu(k),
$$

where $\mathbf{I}_{n}$ denotes a $n \times n$ identity matrix, and

$$
\mu(k)=\left[\begin{array}{l}
-\boldsymbol{\epsilon}(k \mid k-1) \\
\mathbf{r}(k)
\end{array}\right]
$$

is the augmented noise vector comprising of the state and measurement errors. Assuming that the covariance matrix of the augmented noise $\mathrm{E}\left[\mu(k) \mu(k)^{\mathrm{T}}\right]$ is positive definite, we have

$$
\begin{aligned}
& \mathrm{E}\left[\mu(k) \mu(k)^{\mathrm{T}}\right]=\boldsymbol{\Theta}(k) \boldsymbol{\Theta}(k)^{\mathrm{T}} \\
& =\left[\begin{array}{lc}
\boldsymbol{\Theta}_{p}(k \mid k-1) \boldsymbol{\Theta}_{p}(k \mid k-1)^{\mathrm{T}} & \mathbf{0} \\
\mathbf{0} & \boldsymbol{\Theta}_{r}(k) \boldsymbol{\Theta}_{r}(k)^{\mathrm{T}}
\end{array}\right],
\end{aligned}
$$

where $\boldsymbol{\Theta}(k), \boldsymbol{\Theta}_{p}(k \mid k-1)$ and $\boldsymbol{\Theta}_{r}(k)$ are obtained by the Cholesky decomposition of $\mathrm{E}\left[\mu(k) \mu(k)^{\mathrm{T}}\right], \mathbf{P}(k \mid k-1)$ and $\mathbf{R}(k)$, respectively. Multiplying both sides of (16) by $\boldsymbol{\Theta}^{-1}(k)$ gives

$$
\mathbf{d}(k)=\mathbf{W}(k) \mathbf{x}(k)+\mathbf{e}(k),
$$

where

$$
\begin{aligned}
& \mathbf{d}(k)=\boldsymbol{\Theta}^{-1}(k)\left[\begin{array}{l}
\hat{\mathbf{x}}(k \mid k-1) \\
\mathbf{y}(k)
\end{array}\right] \\
& \mathbf{W}(k)=\boldsymbol{\Theta}^{-1}(k)\left[\begin{array}{l}
\mathbf{I}_{n} \\
\mathbf{H}
\end{array}\right] \\
& \mathbf{e}(k)=\boldsymbol{\Theta}^{-1}(k)\left[\begin{array}{l}
-(\mathbf{x}(k)-\hat{\mathbf{x}}(k \mid k-1)) \\
\mathbf{r}(k)
\end{array}\right],
\end{aligned}
$$

with $\mathbf{d}(k)=\left[d_{1}(k), d_{2}(k), \ldots, d_{L}(k)\right]^{\mathrm{T}}, \mathbf{W}(k)=\left[\mathbf{w}_{1}(k), \mathbf{w}_{2}(k)\right.$, $\left.\ldots, \mathbf{w}_{L}(k)\right]^{\mathrm{T}}, \mathbf{e}(k)=\left[e_{1}(k), e_{2}(k), \ldots, e_{L}(k)\right]^{\mathrm{T}}$ and $L=$ $m+n$.

\subsection{Derivation of $M E E-K F$}

Based on (14), the cost function of MEE-KF is given by

$$
J_{L}(\mathbf{x}(k))=\frac{1}{L^{2}} \sum_{i=1}^{L} \sum_{j=1}^{L} G_{\sigma}\left(e_{j}(k)-e_{i}(k)\right)
$$

Then, the optimal solution to $\hat{\mathbf{x}}(k)$ is achieved by maximizing the cost function (23), that is

$$
\begin{aligned}
& \hat{\mathbf{x}}(k)=\underset{\mathbf{x}(k)}{\arg \max _{L}} J_{L}(\mathbf{x}(k)) \\
& =\arg \max _{\mathbf{x}(k)} \frac{1}{L^{2}} \sum_{i=1}^{L} \sum_{j=1}^{L} G_{\sigma}\left(e_{j}(k)-e_{i}(k)\right) .
\end{aligned}
$$

Setting the gradient of the cost function $J_{L}(\mathbf{x}(k))$ regarding $\mathbf{x}(k)$ to zero, we have

$$
\begin{aligned}
& \frac{\partial}{\partial \mathbf{x}(k)} J_{L}(\mathbf{x}(k)) \\
& =\frac{1}{L^{2} \sigma^{2}} \sum_{i=1}^{L} \sum_{j=1}^{L}\left(\begin{array}{l}
{\left[e_{j}(k)-e_{i}(k)\right] G_{\sigma}\left(e_{j}(k)-e_{i}(k)\right)} \\
{\left[\mathbf{w}_{j}(k)-\mathbf{w}_{i}(k)\right]}
\end{array}\right) \\
& =\Gamma_{1}-\Gamma_{2}-\Gamma_{3}+\Gamma_{4}=2 \Gamma_{1}-2 \Gamma_{3} \\
& =\frac{2}{L^{2} \sigma^{2}} \mathbf{W}(k)^{\mathrm{T}} \mathbf{\Psi}(k) \mathbf{e}(k)-\frac{2}{L^{2} \sigma^{2}} \mathbf{W}(k)^{\mathrm{T}} \mathbf{\Phi}(k) \mathbf{e}(k) \\
& =0,
\end{aligned}
$$

where

$$
\begin{aligned}
& \Gamma_{1}=\frac{1}{L^{2} \sigma^{2}} \sum_{i=1}^{L} \sum_{j=1}^{L} e_{j}(k) G_{\sigma}\left(e_{j}(k)-e_{i}(k)\right) \mathbf{w}_{j}(k)^{\mathrm{T}} \\
& \Gamma_{2}=\frac{1}{L^{2} \sigma^{2}} \sum_{i=1}^{L} \sum_{j=1}^{L} e_{i}(k) G_{\sigma}\left(e_{j}(k)-e_{i}(k)\right) \mathbf{w}_{j}(k)^{\mathrm{T}} \\
& \Gamma_{3}=\frac{1}{L^{2} \sigma^{2}} \sum_{i=1}^{L} \sum_{j=1}^{L} e_{j}(k) G_{\sigma}\left(e_{j}(k)-e_{i}(k)\right) \mathbf{w}_{i}(k)^{\mathrm{T}} \\
& \Gamma_{4}=\frac{1}{L^{2} \sigma^{2}} \sum_{i=1}^{L} \sum_{j=1}^{L} e_{i}(k) G_{\sigma}\left(e_{j}(k)-e_{i}(k)\right) \mathbf{w}_{i}(k)^{\mathrm{T}} \\
& {[\mathbf{\Phi}(k)]_{i j}=G_{\sigma}\left(e_{j}(k)-e_{i}(k)\right)} \\
& {[\boldsymbol{\Psi}(k)]_{i j}=\sum_{i=1}^{L} G_{\sigma}\left(e_{j}(k)-e_{i}(k)\right) .}
\end{aligned}
$$

From $(25), \mathbf{x}(k)$ can be solved by a fixed-point iteration:

$$
\begin{aligned}
\hat{\mathbf{x}}(k) & =g(\hat{\mathbf{x}}(k)) \\
& =\left(\mathbf{W}(k)^{\mathrm{T}} \boldsymbol{\Lambda}(k) \mathbf{W}(k)\right)^{-1}\left(\mathbf{W}(k)^{\mathrm{T}} \boldsymbol{\Lambda}(k) \mathbf{d}(k)\right),
\end{aligned}
$$


where

$$
\boldsymbol{\Lambda}(k)=\boldsymbol{\Psi}(k)-\boldsymbol{\Phi}(k)=\left[\begin{array}{cc}
\boldsymbol{\Lambda}_{x}(k) & \boldsymbol{\Lambda}_{y x}(k) \\
\boldsymbol{\Lambda}_{x y}(k) & \boldsymbol{\Lambda}_{y}(k)
\end{array}\right],
$$

where $\boldsymbol{\Lambda}(k) \in \mathbb{R}^{L \times L}$. The explicit expressions of $\boldsymbol{\Lambda}_{x}(k) \in$ $\mathbb{R}^{n \times n}, \boldsymbol{\Lambda}_{x y}(k) \in \mathbb{R}^{m \times n}, \boldsymbol{\Lambda}_{y x}(k) \in \mathbb{R}^{n \times m}$ and $\boldsymbol{\Lambda}_{y}(k) \in$ $\mathbb{R}^{m \times m}$ are

$$
\begin{aligned}
& \boldsymbol{\Lambda}_{x}(k)=\left(\boldsymbol{\Lambda}_{i, j}(k)\right)_{n \times n}=\left(\boldsymbol{\Phi}_{i, j}(k)\right)_{n \times n}-\left(\boldsymbol{\Psi}_{i, j}(k)\right)_{n \times n} \\
& \quad(i=1,2 \cdots n ; j=1,2 \cdots n) \\
& \boldsymbol{\Lambda}_{x y}(k)=\left(\boldsymbol{\Lambda}_{i, j}(k)\right)_{m \times n}=\left(\boldsymbol{\Phi}_{i, j}(k)\right)_{m \times n}-\left(\boldsymbol{\Psi}_{i, j}(k)\right)_{m \times n} \\
& \quad(i=n+1, n+2 \cdots n+m ; j=1,2 \cdots n) \\
& \boldsymbol{\Lambda}_{y x}(k)=\left(\boldsymbol{\Lambda}_{i, j}(k)\right)_{n \times m}=\left(\boldsymbol{\Phi}_{i, j}(k)\right)_{n \times m}-\left(\boldsymbol{\Psi}_{i, j}(k)\right)_{n \times m} \\
& \quad(i=1,2 \cdots n ; j=n+1, n+2 \cdots n+m) \\
& \boldsymbol{\Lambda}_{y}(k)=\left(\boldsymbol{\Lambda}_{i, j}(k)\right)_{m \times m}=\left(\boldsymbol{\Phi}_{i, j}(k)\right)_{m \times m}-\left(\boldsymbol{\Psi}_{i, j}(k)\right)_{m \times m} \\
& \quad(i=n+1, n+2 \cdots n+m ; \\
& \quad j=n+1, n+2 \cdots n+m) .
\end{aligned}
$$

According to Eqs. (20), (21) and (33), we arrive at

$$
\begin{aligned}
& \left(\mathbf{W}(k)^{\mathrm{T}} \boldsymbol{\Lambda}(k) \mathbf{W}(k)\right) \\
& =\left[\boldsymbol{\Theta}_{p}^{-1}(k \mid k-1)^{\mathrm{T}} \boldsymbol{\Lambda}_{x}+\mathbf{H}^{\mathrm{T}} \boldsymbol{\Theta}_{r}^{-1}(k)^{\mathrm{T}} \boldsymbol{\Lambda}_{x y}\right] \boldsymbol{\Theta}_{p}^{-1}(k \mid k-1) \\
& +\left[\boldsymbol{\Theta}_{p}^{-1}(k \mid k-1)^{\mathrm{T}} \boldsymbol{\Lambda}_{y x}+\mathbf{H}^{\mathrm{T}} \boldsymbol{\Theta}_{r}^{-1}(k)^{\mathrm{T}} \boldsymbol{\Lambda}_{y}\right] \boldsymbol{\Theta}_{r}^{-1}(k) \mathbf{H} \\
& \left(\mathbf{W}(k)^{\mathrm{T}} \boldsymbol{\Lambda}(k) \mathbf{D}(k)\right) \\
& =\left[\boldsymbol{\Theta}_{p}^{-1}(k \mid k-1)^{\mathrm{T}} \boldsymbol{\Lambda}_{x}+\mathbf{H}^{\mathrm{T}} \boldsymbol{\Theta}_{r}^{-1}(k)^{\mathrm{T}} \boldsymbol{\Lambda}_{x y}\right] \boldsymbol{\Theta}_{p}^{-1}(k \mid k-1) \\
& \hat{\mathbf{x}}(k \mid k-1)+\left[\boldsymbol{\Theta}_{p}^{-1}(k \mid k-1)^{\mathrm{T}} \boldsymbol{\Lambda}_{y x}+\mathbf{H}^{\mathrm{T}} \boldsymbol{\Theta}_{r}^{-1}(k)^{\mathrm{T}} \boldsymbol{\Lambda}_{y}\right] \\
& \boldsymbol{\Theta}_{r}^{-1}(k) \mathbf{y}(k) .
\end{aligned}
$$

By (38), the Eq. (32) can be rewritten as

$$
\begin{aligned}
\hat{\mathbf{x}}(k) & =\left(\boldsymbol{\Omega}_{1}+\boldsymbol{\Omega}_{2} \boldsymbol{\Omega}_{3}\right)^{-1}\left(\boldsymbol{\Omega}_{1} \hat{\mathbf{x}}(k \mid k-1)+\boldsymbol{\Omega}_{2} \mathbf{y}(k)\right) \\
& =\left(\boldsymbol{\Omega}_{1}+\boldsymbol{\Omega}_{2} \boldsymbol{\Omega}_{3}\right)^{-1} \boldsymbol{\Omega}_{1} \hat{\mathbf{x}}(k \mid k-1) \\
& +\left(\boldsymbol{\Omega}_{1}+\boldsymbol{\Omega}_{2} \boldsymbol{\Omega}_{3}\right)^{-1} \boldsymbol{\Omega}_{2} \mathbf{y}(k),
\end{aligned}
$$

where

$$
\begin{aligned}
& \boldsymbol{\Omega}_{1}=\left[\boldsymbol{\Theta}_{p}^{-1}(k \mid k-1)^{\mathrm{T}} \boldsymbol{\Lambda}_{x}+\mathbf{H}^{\mathrm{T}} \boldsymbol{\Theta}_{r}^{-1}(k)^{\mathrm{T}} \boldsymbol{\Lambda}_{x y}\right] \boldsymbol{\Theta}_{p}^{-1}(k \mid k-1) \\
& \boldsymbol{\Omega}_{2}=\left[\boldsymbol{\Theta}_{p}^{-1}(k \mid k-1)^{\mathrm{T}} \boldsymbol{\Lambda}_{y x}+\mathbf{H}^{\mathrm{T}} \boldsymbol{\Theta}_{r}^{-1}(k)^{\mathrm{T}} \boldsymbol{\Lambda}_{y}\right] \boldsymbol{\Theta}_{r}^{-1}(k) \\
& \boldsymbol{\Omega}_{3}=\mathbf{H} \text {. }
\end{aligned}
$$

By using the matrix inversion lemma

$$
\begin{aligned}
& (\mathbf{A}+\mathbf{B C D})^{-1} \\
& =\mathbf{A}^{-1}-\mathbf{A}^{-1} \mathbf{B}\left(\mathbf{C}^{-1}+\mathbf{D A}^{-1} \mathbf{B}\right)^{-1} \mathbf{D} \mathbf{A}^{-1},
\end{aligned}
$$

with the identifications

$$
\boldsymbol{\Omega}_{1} \rightarrow \mathbf{A}, \boldsymbol{\Omega}_{2} \rightarrow \mathbf{B}, \mathbf{I}_{L} \rightarrow \mathbf{C}, \boldsymbol{\Omega}_{3} \rightarrow \mathbf{D},
$$

one can reformulate (39) as

$$
\hat{\mathbf{x}}(k)=\hat{\mathbf{x}}(k \mid k-1)+\overline{\mathbf{K}}(k)(\mathbf{y}(k)-\mathbf{H} \hat{\mathbf{x}}(k \mid k-1)),
$$

where

$$
\begin{aligned}
& \overline{\mathbf{K}}(k)=\left[\begin{array}{l}
\overline{\mathbf{P}}(k \mid k-1)+\mathbf{H}^{\mathrm{T}} \overline{\mathbf{P}}_{x y}(k \mid k-1) \\
+\left(\overline{\mathbf{P}}_{y x}(k \mid k-1)+\mathbf{H}^{\mathrm{T}} \overline{\mathbf{R}}(k)\right) \mathbf{H}
\end{array}\right]^{-1} \\
& \quad \times\left(\overline{\mathbf{P}}_{y x}(k \mid k-1)+\mathbf{H}^{\mathrm{T}} \overline{\mathbf{R}}(k)\right) \\
& \overline{\mathbf{P}}(k \mid k-1)=\boldsymbol{\Theta}_{p}^{-1}(k \mid k-1)^{\mathrm{T}} \boldsymbol{\Lambda}_{x}(k) \boldsymbol{\Theta}_{p}^{-1}(k \mid k-1) \\
& \overline{\mathbf{P}}_{x y}(k \mid k-1)=\boldsymbol{\Theta}_{r}^{-1}(k)^{\mathrm{T}} \boldsymbol{\Lambda}_{x y}(k) \boldsymbol{\Theta}_{p}^{-1}(k \mid k-1) \\
& \overline{\mathbf{P}}_{y x}(k \mid k-1)=\boldsymbol{\Theta}_{p}^{-1}(k \mid k-1)^{\mathrm{T}} \boldsymbol{\Lambda}_{y x}(k) \boldsymbol{\Theta}_{r}^{-1}(k) \\
& \overline{\mathbf{R}}(k)=\boldsymbol{\Theta}_{r}^{-1}(k)^{\mathrm{T}} \boldsymbol{\Lambda}_{y}(k) \boldsymbol{\Theta}_{r}^{-1}(k) .
\end{aligned}
$$

Then, the posterior covariance matrix can be updated by

$$
\begin{aligned}
\mathbf{P}(k)= & {[\mathbf{I}-\overline{\mathbf{K}}(k) \mathbf{H}] \mathbf{P}(k \mid k-1)[\mathbf{I}-\overline{\mathbf{K}}(k) \mathbf{H}]^{\mathrm{T}} } \\
& +\overline{\mathbf{K}}(k) \mathbf{R}(k) \overline{\mathbf{K}}(k)^{\mathrm{T}} .
\end{aligned}
$$

With the above derivations, the proposed MEE-KF algorithm can be summarized as Algorithm 1.

\section{Computational Complexity}

This section provides the comparison of the computational complexities of $\mathrm{KF}$, maximum correntropy Kalman filter (MCKF) [15] and MEE-KF in terms of the floating point operations.

The KF updates with Eqs. (6)-(10), and the corresponding floating point operations are given in Table 1. From Table 1, we can conclude that the computational complexity of $\mathrm{KF}$ is

$$
C_{K F}=8 n^{3}+10 n^{2} m-n^{2}+6 n m^{2}-n+O\left(m^{3}\right) .
$$

According to [15], the computational complexity of $\mathrm{MCKF}$ is

$$
\begin{aligned}
C_{M C K F} & =(2 T+8) n^{3}+(4 T+6) n^{2} m+(2 T-1) n^{2} \\
& +(4 T+2) n m^{2}+(3 T-1) n m+(4 T-1) n \\
& +2 T m^{3}+2 T m+T O\left(n^{3}\right)+2 T O\left(m^{3}\right), \quad(56)
\end{aligned}
$$


Algorithm 1 Minimum Error Entropy Kalman Filter (MEE-KF)

Step 1: Initialize the state priori estimate $\hat{\mathbf{x}}(1 \mid 0)$ and state prediction error covariance matrix $\mathbf{P}(1 \mid 0)$; set a proper kernel size $\sigma$ and a small positive number $\varepsilon$.

Step 2: Use Eqs. (6) and (7) to obtain $\hat{\mathbf{x}}(k \mid k-1)$ and $\mathbf{P}(k \mid k-1)$, respectively; use the Cholesky decomposition of $\boldsymbol{\Theta}(k)$ to obtain $\boldsymbol{\Theta}_{p}(k \mid k-1)$ and $\boldsymbol{\Theta}_{r}(k)$; use Eqs. (20) and (21) to obtain $\mathbf{d}(k)$ and $\mathbf{W}(k-1)$, respectively. Step 3: Let $t=1$ and $\hat{\mathbf{x}}(k)_{0}=\hat{\mathbf{x}}(k \mid k-1)$, where $\hat{\mathbf{x}}(k)_{t}$ denotes the estimated state at the fixed-point iteration $t$.

Step 4: Use available measurements $\{\mathbf{y}(k)\}_{k=1}^{N}$ to update:

$$
\hat{\mathbf{x}}(k)_{t}=\hat{\mathbf{x}}(k \mid k-1)+\widetilde{\mathbf{K}}(k)[\mathbf{y}(k)-\mathbf{H} \hat{\mathbf{x}}(k \mid k-1)]
$$

with

$$
\begin{aligned}
& \widetilde{\mathbf{e}}_{i}(k)=\mathbf{d}_{i}(k)-\mathbf{w}(k) \hat{\mathbf{x}}(k)_{t-1} \\
& \widetilde{\boldsymbol{\Lambda}}(k)=\widetilde{\boldsymbol{\Psi}}(k)-\widetilde{\boldsymbol{\Phi}}(k)=\left[\begin{array}{cc}
\widetilde{\boldsymbol{\Lambda}}_{x}(k) & \widetilde{\boldsymbol{\Lambda}}_{y x}(k) \\
\widetilde{\boldsymbol{\Lambda}}_{x y}(k) & \widetilde{\boldsymbol{\Lambda}}_{y}(k)
\end{array}\right] \\
& \widetilde{\mathbf{P}}(k \mid k-1)=\boldsymbol{\Theta}_{p}^{-1}(k \mid k-1)^{\mathrm{T}} \widetilde{\boldsymbol{\Lambda}}_{x}(k) \boldsymbol{\Theta}_{p}^{-1}(k \mid k-1) \\
& \widetilde{\mathbf{P}}_{x y}(k \mid k-1)=\boldsymbol{\Theta}_{r}^{-1}(k)^{\mathrm{T}} \widetilde{\boldsymbol{\Lambda}}_{x y}(k) \boldsymbol{\Theta}_{p}^{-1}(k \mid k-1) \\
& \widetilde{\mathbf{P}}_{y x}(k \mid k-1)=\boldsymbol{\Theta}_{p}^{-1}(k \mid k-1)^{\mathrm{T}} \widetilde{\boldsymbol{\Lambda}}_{y x}(k) \boldsymbol{\Theta}_{r}^{-1}(k) \\
& \widetilde{\mathbf{R}}(k)=\boldsymbol{\Theta}_{r}^{-1}(k)^{\mathrm{T}} \widetilde{\boldsymbol{\Lambda}}_{y}(k) \boldsymbol{\Theta}_{r}^{-1}(k) \\
& \widetilde{\mathbf{K}}(k)=\left[\begin{array}{l}
\widetilde{\mathbf{P}}(k \mid k-1)+\mathbf{H}^{\mathrm{T}} \widetilde{\mathbf{P}}_{x y}(k \mid k-1) \\
+\left(\widetilde{\mathbf{P}}_{y x}(k \mid k-1)+\mathbf{H}^{\mathrm{T}} \widetilde{\mathbf{R}}(k)\right) \mathbf{H}
\end{array}\right]^{-1} \\
& \times\left(\widetilde{\mathbf{P}}_{y x}(k \mid k-1)+\mathbf{H}^{\mathrm{T}} \widetilde{\mathbf{R}}(k)\right)
\end{aligned}
$$

Step 5: Compare $\hat{\mathbf{x}}(k)_{t}$ and $\hat{\mathbf{x}}(k)_{t-1}$

$$
\frac{\left\|\hat{\mathbf{x}}(k)_{t}-\hat{\mathbf{x}}(k)_{t-1}\right\|}{\left\|\hat{\mathbf{x}}(k)_{t-1}\right\|} \leq \varepsilon .
$$

If the above condition holds, set $\hat{\mathbf{x}}(k)=\hat{\mathbf{x}}(k)_{t}$ and con-

\begin{tabular}{|c|c|c|}
\hline Equation & $\begin{array}{l}\text { Addition/subtraction } \\
\text { and multiplication }\end{array}$ & $\begin{array}{l}\text { Division, matrix } \\
\text { inversion, Cholesky } \\
\text { decomposition and } \\
\text { exponentiation }\end{array}$ \\
\hline (6) & $2 n^{2}-n$ & 0 \\
\hline (7) & $4 n^{3}-n^{2}$ & 0 \\
\hline (8) & $4 n m$ & 0 \\
\hline (9) & $4 n^{2} m+4 n m^{2}-3 n m$ & $O\left(m^{3}\right)$ \\
\hline (10) & $\begin{array}{l}4 n^{3}+6 n^{2} m-2 n^{2}+ \\
2 n m^{2}-n m\end{array}$ & 0 \\
\hline$(45)$ & $4 n m$ & $O\left(m^{2}\right)+O(m n)$ \\
\hline$(46)$ & $2 n$ & 0 \\
\hline$(47)$ & $3(m+n)^{3}+(m+n)^{2}$ & $(m+n)^{2}$ \\
\hline$(48)$ & $4 n^{3}-2 n^{2}$ & $n+O\left(n^{3}\right)$ \\
\hline (49) & $2 n^{2} m+2 n m^{2}-2 n m$ & 0 \\
\hline$(50)$ & $2 n^{2} m+2 n m^{2}-2 n m$ & 0 \\
\hline$(51)$ & $4 m^{3}-2 m^{2}$ & $m+O\left(m^{3}\right)$ \\
\hline$(52)$ & $6 n^{2} m+2 n m^{2}-n m$ & $O\left(m^{3}\right)$ \\
\hline$(54)$ & $\begin{array}{l}4 n^{3}+6 n^{2} m-2 n^{2}+ \\
2 n m^{2}-n m\end{array}$ & 0 \\
\hline
\end{tabular}
tinue to Step 6. Otherwise, $t+1 \rightarrow t$, and return to Step 4.

Step 6: Update $k+1 \rightarrow k$ and the posterior error covariance matrix by

$$
\begin{aligned}
\mathbf{P}(k)= & {[\mathbf{I}-\widetilde{\mathbf{K}}(k) \mathbf{H}] \mathbf{P}(k \mid k-1)[\mathbf{I}-\widetilde{\mathbf{K}}(k) \mathbf{H}]^{\mathrm{T}} } \\
& +\widetilde{\mathbf{K}}(k) \mathbf{R}(k) \widetilde{\mathbf{K}}^{\mathrm{T}}(k),
\end{aligned}
$$

and return to Step 2.
Table 1

Computational Complexities of Some Equations

where $T$ denotes the fixed-point iteration number, which is relatively small in general as shown in simulations in Section VII.

The updates of MEE-KF involve Eqs. (6), (7), (45)-(52) and (54), and the corresponding floating point operations are shown in Table 1 . According to Table 1, the computational complexity of MEE-KF is

$$
\begin{aligned}
C_{M E E-K F} & =(7 T+8) n^{3}+(7 T) m^{3}+(19 T+6) n^{2} m-n^{2} \\
& +(15 T+2) n m^{2}+T O\left(n^{3}\right)+T O(m n) \\
& +T m+(5 T-1) n+(7 T-1) n m \\
& +T O\left(m^{2}\right)+2 T O\left(m^{3}\right) .
\end{aligned}
$$

The MEE-KF has an additional computational burden induced by the error entropy functions in comparison to $\mathrm{KF}$, and has a slightly higher computational complexity than MCKF. In the sense of order of magnitude, the computational complexities of the MEE-KF, MCKF and $\mathrm{KF}$ have no significant difference.

\section{Convergence Issue}

This section provides a sufficient condition to ensure the convergence of the fixed point iterations in MEE-KF, where the proof is similar to [35] and thus will not be provided here. 
First, from Eq. (32), we can rewrite

$$
g(\mathbf{x}(k))=\mathbf{M}_{\mathbf{w w}}^{-1} \mathbf{M}_{\mathbf{d w}}
$$

with $\mathbf{M}_{\mathbf{w w}}^{-1}=\sum_{i=1}^{L} \sum_{j=1}^{L} \begin{aligned} & G_{\sigma}\left(e_{j}(k)-e_{i}(k)\right)\left[\mathbf{w}_{j}(k)-\mathbf{w}_{i}(k)\right] \\ & \times\left[\mathbf{w}_{j}(k)-\mathbf{w}_{i}(k)\right]^{\mathrm{T}}\end{aligned}$

and $\mathbf{M}_{\mathbf{d w}}=\sum_{i=1}^{L} \sum_{j=1}^{L} \begin{aligned} & \left.G_{\sigma}\left(e_{j}(k)-e_{i}(k)\right)\right]\left[d_{j}(k)-d_{i}(k)\right] \\ & \left.\times \mathbf{w}_{i}(k)\right]\end{aligned}$.

Thus, a $n \times n$ Jacobian matrix of $g(\mathbf{x}(k))$ with respect to $\mathbf{x}(k)$ gives

$$
\begin{aligned}
& \nabla_{\mathbf{x}(k)} g(\mathbf{x}(k))=\frac{\partial}{\partial \mathbf{x}(k)} \mathbf{M}_{\mathbf{w w}}^{-1} \mathbf{M}_{\mathbf{d w}} \\
& =\left[\begin{array}{ll}
\frac{\partial g(\mathbf{x}(k))}{\partial \mathbf{x}_{1}(k)} & \left.\frac{\partial g(\mathbf{x}(k))}{\partial \mathbf{x}_{2}(k)} \cdots \frac{\partial g(\mathbf{x}(k))}{\partial \mathbf{x}_{L}}\right]
\end{array}\right] \\
& =-\frac{\mathbf{M}_{\mathbf{w w}}^{-1}}{2 L^{2} \sigma^{2}}\left(\sum_{i=1}^{L} \sum_{j=1}^{L} \xi_{1}(k)\left[\mathbf{w}_{j}(k)-\mathbf{w}_{j}(k)-\mathbf{w}_{i}(k)\right]^{\mathrm{T}}\right) g(\mathbf{x}(k)) \\
& +\frac{\mathbf{M}_{\mathbf{w w}}^{-1}}{2 L^{2} \sigma^{2}}\left(\sum_{i=1}^{L} \sum_{j=1}^{L} \xi_{1}(k)\left[d_{j}(k)-d_{i}(k)\right]\right),
\end{aligned}
$$

with $\xi_{1}(k)=\left(e_{j}(k)-e_{i}(k)\right)\left(\mathbf{w}_{j}(k)-\mathbf{w}_{i}(k)\right) G_{\sigma}(e(j)-$ $e(i))$. Define $\|\cdot\|_{p}$ as an $l_{p}$-norm $(p \geq 1)$ of a vector or an induced norm of a matrix as $\|\mathbf{A}\|_{p}=$ $\max _{\| \mathbf{W}||_{p} \neq 0}\|\mathbf{A W}\|_{p} /\|\mathbf{W}\|_{p}$. According to the proof in [35], the following theorem holds.

Theorem 1 If the kernel size satisfies $\sigma \geq \max \left\{\sigma_{1}, \sigma_{2}\right\}$, we have

$$
\left\{\begin{array}{l}
\|g(\boldsymbol{x}(k))\|_{1} \leq \beta \\
\left\|\nabla_{x(k)} g(\boldsymbol{x}(k))\right\|_{1} \leq \alpha<1,
\end{array}\right.
$$

where $\beta>\rho=\frac{\sqrt{n} \sum_{i=1}^{L} \sum_{j=1}^{L} \times\left[d_{j}(k)-d_{i}(k)\right]}{\lambda_{\min }\left[\sum_{i=1}^{L} \sum_{j=1}^{L}\left[\boldsymbol{w}_{j}(k)-\boldsymbol{w}_{i}(k)\right]\right.}$; $\lambda_{\min }$ is the minimum eigenvalue of the matrix $\boldsymbol{M}_{\boldsymbol{w} w}$; $\sigma_{1}$ and $\sigma_{2}$ are the solutions of $\|g(\boldsymbol{x}(k))\|_{1}=\beta$ and $\left\|\nabla_{x(k)} g(\boldsymbol{x}(k))\right\|_{1}=\alpha$, respectively, i.e.,

$$
\|g(\boldsymbol{x}(k))\|_{1}=\frac{\sqrt{n} \sum_{i=1}^{L} \sum_{j=1}^{L} \times\left[d_{j}(k)-d_{i}(k)\right]}{\lambda_{\min }\left[\sum_{i=1}^{L} \sum_{j=1}^{L} \xi_{2}(k)\left[\boldsymbol{w}_{j}(k)-\boldsymbol{w}_{i}(k)\right]\right.}
$$

with $\xi_{2}(k)=G_{\sigma}\left(\beta\left\|\boldsymbol{w}_{j}(k)-\boldsymbol{w}_{i}(k)\right\|_{1}+\left|d_{j}(k)-d_{i}(k)\right|\right)$, and

$$
\left\|\nabla_{x(k)} g(\boldsymbol{x}(k))\right\|_{1}=\frac{\gamma \sqrt{n}}{\sigma^{2} \lambda_{\min }\left[\sum_{i=1}^{L} \sum_{j=1}^{L} \times\left[\begin{array}{l}
\xi_{2}(k)\left[\boldsymbol{w}_{j}(k)-\boldsymbol{w}_{i}(k)\right] \\
\left.\boldsymbol{w}_{i}(k)\right]^{T}
\end{array}\right]\right.}
$$

$$
\begin{aligned}
& \left(\beta\left\|\boldsymbol{w}_{j}(k)-\boldsymbol{w}_{i}(k)\right\|_{1}+\left|d_{j}(k)-d_{i}(k)\right|\right) \\
\text { with } \gamma=\sum_{i=1}^{L} \sum_{j=1}^{L} & \left\|\boldsymbol{w}_{j}(k)-\boldsymbol{w}_{i}(k)\right\|_{1}\left(\beta \|\left[\boldsymbol{w}_{j}(k)-\boldsymbol{w}_{i}(k)\right]\right. \\
& {\left[\boldsymbol{w}_{j}(k)-\boldsymbol{w}_{i}(k)\right]^{T} \|_{1}+\left[d_{j}(k)-d_{i}(k)\right] } \\
& \left.\left\|\boldsymbol{w}_{j}(k)-\boldsymbol{w}_{i}(k)\right\|_{1}\right)
\end{aligned}
$$

According to Theorem 1 and Banach Fixed Point Theorem, the fixed-point algorithm induced by (32) can be guaranteed to converge to a unique fixed point in the range $\mathbf{x}(k) \in\left\{\|\mathbf{x}(k)\|_{1} \leq \beta\right\}$ provided that the kernel size $\sigma$ is larger than a certain value and an initial state vector satisfies $\left\|\mathbf{x}(k)_{0}\right\|_{1} \leq \beta$.

\section{Minimum Error Entropy Extended Kalman Filter}

For a nonlinear system, the state and measurement equations can be described by

$$
\begin{aligned}
& \mathbf{x}(k)=\mathbf{f}(\mathbf{x}(k-1))+\mathbf{q}(k-1) \\
& \mathbf{y}(k)=\mathbf{h}(\mathbf{x}(k))+\mathbf{r}(k)
\end{aligned}
$$

where $\mathbf{f}(\cdot)$ and $\mathbf{h}(\cdot)$ are the state-transition function and measurement (observation) function, respectively. The EKF is an important tool for dealing with the state estimation of a nonlinear system via approximating the nonlinear system by linear model. In EKF, the nonlinear functions $\mathbf{f}(\mathbf{x}(k-1))$ and $\mathbf{h}(\mathbf{x}(k))$ can be approximated by the first-order Taylor series expansion at $\hat{\mathbf{x}}(k)$ and $\hat{\mathbf{x}}(k \mid k-1)$, i.e.,

$$
\begin{aligned}
& \mathbf{f}(\mathbf{x}(k-1)) \approx \mathbf{f}(\hat{\mathbf{x}}(k-1))+\mathbf{F}(k-1)(\mathbf{x}(k-1)-\hat{\mathbf{x}}(k-1)) \\
& \mathbf{h}(\mathbf{x}(k)) \approx \mathbf{h}(\hat{\mathbf{x}}(k \mid k-1))+\mathbf{H}(k)(\mathbf{x}(k)-\hat{\mathbf{x}}(k \mid k-1)),
\end{aligned}
$$

where $\mathbf{F}(k-1)$ and $\mathbf{H}(k)$ are the Jacobian matrices of $\mathbf{f}(\cdot)$ and $\mathbf{h}(\cdot)$, i.e.,

$$
\begin{aligned}
& \mathbf{F}(k-1)=\left.\frac{\partial \mathbf{f}(\mathbf{x}(k-1))}{\partial \mathbf{x}}\right|_{\mathbf{x}=\hat{\mathbf{x}}(k)} \\
& \mathbf{H}(k)=\left.\frac{\partial \mathbf{h}(\mathbf{x}(k))}{\partial \mathbf{x}}\right|_{\mathbf{x}=\hat{\mathbf{x}}(k \mid k-1)} .
\end{aligned}
$$


By substituting (65) and (66) into (63) and (64), the prediction and update equations of EKF can be obtained:

$$
\begin{aligned}
& \hat{\mathbf{x}}(k \mid k-1)=\mathbf{f}(\hat{\mathbf{x}}(k-1)) \\
& \mathbf{P}(k \mid k-1)=\mathbf{F}(k-1) \mathbf{P}(k-1) \mathbf{F}^{\mathrm{T}}(k-1)+\mathbf{Q}(k-1)
\end{aligned}
$$

$$
\mathbf{K}(k)=\mathbf{P}(k \mid k-1) \mathbf{H}^{\mathrm{T}}(k)\left[\mathbf{H}(k) \mathbf{P}(k \mid k-1) \mathbf{H}^{\mathrm{T}}(k)+\mathbf{R}(k)\right]^{-1}
$$

$$
\hat{\mathbf{x}}(k)=\hat{\mathbf{x}}(k \mid k-1)+\mathbf{K}(k)[\mathbf{y}(k)-\mathbf{h}(\hat{\mathbf{x}}(k \mid k-1))]
$$$$
\mathbf{P}(k)=[\mathbf{I}-\mathbf{K}(k) \mathbf{H}(k)] \mathbf{P}(k \mid k-1)[\mathbf{I}-\mathbf{K}(k) \mathbf{H}(k)]^{\mathrm{T}}
$$$$
+\mathbf{K}(k) \mathbf{R}(k) \mathbf{K}^{\mathrm{T}}(k),
$$

where $\mathbf{K}(k)$ is the gain of EKF. Similar to MEE-KF and maximum correntropy extended Kalman filter (MCEKF) [25], the augmented model of MEE-EKF should be established. By substituting (66) into (64), the measurement equation is approximated by

$$
\begin{aligned}
\mathbf{y}(k) & \approx \mathbf{h}(\hat{\mathbf{x}}(k \mid k-1)) \\
& +\mathbf{H}(k)(\mathbf{x}(k)-\hat{\mathbf{x}}(k \mid k-1))+\mathbf{r}(k) .
\end{aligned}
$$

Then, replacing $\mathbf{y}(k)$ in (16) with that in (74), one can obtain the following augmented model:

$$
\begin{array}{r}
{\left[\begin{array}{l}
\hat{\mathbf{x}}(k \mid k-1) \\
\mathbf{y}(k)-\mathbf{h}(\hat{\mathbf{x}}(k \mid k-1))+\mathbf{H}(k) \hat{\mathbf{x}}(k \mid k-1)
\end{array}\right]} \\
=\left[\begin{array}{l}
\mathbf{I}_{n} \\
\mathbf{H}(k)
\end{array}\right] \mathbf{x}(k)+\mu(k),
\end{array}
$$

where $\mu(k)$ has the same form with (17). Similar to (19), we have

$$
\mathbf{d}(k)=\mathbf{W}(k) \mathbf{x}(k)+\mathbf{e}(k),
$$

where

$\mathbf{d}(k)=\Theta^{-1}(k)\left[\begin{array}{l}\hat{\mathbf{x}}(k \mid k-1) \\ \mathbf{y}(k)-\mathbf{h}(\hat{\mathbf{x}}(k \mid k-1))+\mathbf{H}(k) \hat{\mathbf{x}}(k \mid k-1)\end{array}\right]$

$\mathbf{W}(k)=\Theta^{-1}(k)\left[\begin{array}{l}\mathbf{I}_{n} \\ \mathbf{H}(k)\end{array}\right]$.

The forms of $\boldsymbol{\Theta}(k)$ and $\mathbf{e}(k)$ are the same as Eqs. (18) and (22), respectively. Therefore, using the Eq. (76) and the derivation similar to that of MEE-KF, one can obtain the MEE-EKF, as provided in Algorithm 2.

\section{Experimental Results}

In this section, the performances of the proposed MEE$\mathrm{KF}$ and MEE-EKF are demonstrated in different scenarios through three examples, i.e., land vehicle navigation,
Algorithm 2 Minimum Error Entropy Extended Kalman Filter (MEE-EKF)

Step 1: Similar to the Step 1 in Algorithm 1.

Step 2: Use Eqs. (67), (69), and (70) to obtain $\hat{\mathbf{x}}(k \mid k-1)$ and $\mathbf{P}(k \mid k-1)$; use the Cholesky decomposition of $\boldsymbol{\Theta}(k)$ to obtain $\boldsymbol{\Theta}_{p}(k \mid k-1)$ and $\boldsymbol{\Theta}_{r}(k)$; use Eqs. (77) and (78) to obtain $\mathbf{d}(k)$ and $\mathbf{W}(k-1)$, respectively.

Step 3: Similar to the Step 3 in Algorithm 1.

Step 4: Use the available measurements $\{\mathbf{y}(k)\}_{k=1}^{N}$ to update:

$$
\begin{aligned}
\hat{\mathbf{x}}(k)_{t} & =\hat{\mathbf{x}}(k \mid k-1)+\widetilde{\mathbf{K}}(k)[\mathbf{y}(k)-\mathbf{h}(\hat{\mathbf{x}}(k \mid k-1))] \\
\widetilde{\mathbf{e}}_{i}(k) & =\mathbf{d}_{i}(k)-\mathbf{w}(k) \hat{\mathbf{x}}(k)_{t-1} \\
\widetilde{\mathbf{K}}(k) & =\left[\begin{array}{l}
\widetilde{\mathbf{P}}(k \mid k-1)+\mathbf{H}^{\mathrm{T}}(k) \widetilde{\mathbf{P}}_{x y}(k \mid k-1) \\
+\left(\widetilde{\mathbf{P}}_{y x}(k \mid k-1)+\mathbf{H}^{\mathrm{T}}(k) \widetilde{\mathbf{R}}(k)\right) \mathbf{H}(k)
\end{array}\right]^{-1} \\
& \times\left(\widetilde{\mathbf{P}}_{y x}(k \mid k-1)+\mathbf{H}^{\mathrm{T}}(k) \widetilde{\mathbf{R}}(k)\right),
\end{aligned}
$$

where the forms of $\widetilde{\boldsymbol{\Lambda}}(k), \widetilde{\mathbf{P}}(k \mid k-1), \widetilde{\mathbf{P}}_{x y}(k \mid k-1)$, $\widetilde{\mathbf{P}}_{y x}(k \mid k-1)$ and $\widetilde{\mathbf{R}}(k)$ are the same as Eqs. (47)-(51), respectively.

Step 5: Similar to the Step 1 in Algorithm 1.

Step 6: Update $k+1 \rightarrow k$ and the posterior covariance matrix:

$$
\begin{aligned}
\mathbf{P}(k)= & {[\mathbf{I}-\widetilde{\mathbf{K}}(k) \mathbf{H}(k)] \mathbf{P}(k \mid k-1)[\mathbf{I}-\widetilde{\mathbf{K}}(k) \mathbf{H}(k)]^{\mathrm{T}} } \\
& +\widetilde{\mathbf{K}}(k) \mathbf{R}(k) \widetilde{\mathbf{K}}^{\mathrm{T}}(k)
\end{aligned}
$$

and return to Step 2 .

tracking of autonomous driving $[43,44]$ and prediction of infectious disease epidemics [45]. The results are compared with $\mathrm{KF}$ and MCKF in linear model, and EKF and MCEKF in nonlinear model. The performance evaluation index is defined by the mean square error (MSE):

$$
\mathrm{MSE}=\frac{1}{N} \sum_{k=1}^{N}\|\mathbf{x}(k)-\hat{\mathbf{x}}(k)\|_{1}
$$

where $\hat{\mathbf{x}}(k)$ is the estimate of $\mathbf{x}(k)$, and $N$ is the number of samples. In the simulations, the MSE is computed by averaging over 100 independent Monte Carlo runs.

\subsection{Land Vehicle Navigation}

In the first example, we assume a linear land vehicle navigation problem to illustrate the performance of MEEKF. 
Table 2

Estimation Results of Different Algorithms Under Different Noises

\begin{tabular}{llllll}
\hline Noises & Algorithms & MSE of x1 & MSE of x2 & MSE of x3 & MSE of x4 \\
\hline Gaussian noise & KF & $0.0762 \pm 0.0087$ & $0.0762 \pm 0.0026$ & $0.0626 \pm 0.0089$ & $0.0593 \pm 0.0027$ \\
& MCKF & $0.0762 \pm 0.0074$ & $0.0762 \pm 0.0027$ & $0.0627 \pm 0.0080$ & $0.0593 \pm 0.0059$ \\
& MEE-KF & $0.0791 \pm 0.0042$ & $0.0789 \pm 0.0045$ & $0.0711 \pm 0.0010$ & $0.0725 \pm 0.0085$ \\
Gaussian noise & KF & $0.5011 \pm 0.0502$ & $0.4868 \pm 0.0783$ & $0.1595 \pm 0.0812$ & $0.1396 \pm 0.0189$ \\
with outliers & MCKF & $0.3803 \pm 0.0524$ & $0.3655 \pm 0.0785$ & $0.1495 \pm 0.0867$ & $0.1295 \pm 0.0192$ \\
& MEE-KF & $\mathbf{0 . 2 7 8 5} \pm \mathbf{0 . 0 3 6 8}$ & $\mathbf{0 . 1 7 9 4} \pm \mathbf{0 . 0 4 7 8}$ & $\mathbf{0 . 1 3 7 7} \pm \mathbf{0 . 0 4 6 7}$ & $\mathbf{0 . 1 1 5 5} \pm \mathbf{0 . 0 2 4 5}$ \\
mixture Gaussian & KF & $0.4903 \pm 0.0887$ & $0.4840 \pm 0.0375$ & $0.1572 \pm 0.0363$ & $0.1292 \pm 0.0889$ \\
noise & MCKF & $0.4452 \pm 0.0005$ & $0.4376 \pm 0.0365$ & $0.1527 \pm 0.0770$ & $0.1348 \pm 0.0699$ \\
& MEE-KF & $\mathbf{0 . 2 7 1 4} \pm \mathbf{0 . 0 3 1 4}$ & $\mathbf{0 . 1 7 9 6} \pm \mathbf{0 . 0 9 6 2}$ & $\mathbf{0 . 1 3 6 4} \pm \mathbf{0 . 0 7 0}$ & $\mathbf{0 . 1 1 6 1} \pm \mathbf{0 . 0 3 6 8}$ \\
mixture Gaussian & KF & $1.1973 \pm 0.0369$ & $1.1918 \pm 0.0468$ & $0.3911 \pm 0.0126$ & $0.3640 \pm 0.0469$ \\
noise with outliers & MCKF & $0.8420 \pm 0.0789$ & $0.8256 \pm 0.0546$ & $0.3604 \pm 0.0345$ & $0.3330 \pm 0.0549$ \\
& MEE-KF & $\mathbf{0 . 6 0 8 7} \pm \mathbf{0 . 0 5 2 4}$ & $\mathbf{0 . 4 9 9 8} \pm \mathbf{0 . 0 4 7 9}$ & $\mathbf{0 . 3 2 2 5} \pm \mathbf{0 . 0 4 2 5}$ & $\mathbf{0 . 3 1 6 1} \pm \mathbf{0 . 0 2 4 5}$ \\
\hline
\end{tabular}

\subsubsection{Model}

The state and measurement equations are described by

$$
\begin{aligned}
& \mathbf{x}(k)=\left[\begin{array}{cccc}
1 & 0 & \Delta T & 0 \\
0 & 1 & 0 & \Delta T \\
0 & 0 & 1 & 0 \\
0 & 0 & 0 & 1
\end{array}\right] \mathbf{x}(k-1)+\mathbf{q}(k-1) \\
& \mathbf{y}(k)=\left[\begin{array}{cccc}
-1 & 0 & -1 & 0 \\
0 & -1 & 0 & -1
\end{array}\right] \mathbf{x}(k-1)+\mathbf{r}(k),
\end{aligned}
$$

where $\mathbf{x}(k)=\left[\mathrm{x}_{1}(k) \mathrm{x}_{2}(k) \mathrm{x}_{3}(k) \mathrm{x}_{4}(k)\right]^{\mathrm{T}}$ is the state vector with components being the north position, the east position, the north velocity and the east velocity. The variables $\theta=\pi / 3$ and $\Delta T=0.3$ are the direction, and time interval of vehicle, respectively. The real state $\mathbf{x}(0)$, the prior estimate $\hat{\mathbf{x}}(1 \mid 0)$ and the corresponding error covariance matrix $\mathbf{P}(1 \mid 0)$ are initialized by

$$
\begin{aligned}
& \mathbf{x}(0)=\left[\begin{array}{llll}
0 & 0 & 10 \tan \theta & 10
\end{array}\right]^{\mathrm{T}} \\
& \hat{\mathbf{x}}(1 \mid 0)=\left[\begin{array}{llll}
1 & 1 & 1 & 1
\end{array}\right]^{\mathrm{T}} \\
& \mathbf{P}(1 \mid 0)=\operatorname{diag}\left[\begin{array}{llll}
900 & 900 & 4 & 4
\end{array}\right] \text {. }
\end{aligned}
$$

In the dynamic system, the process noises are assumed to be of the Gaussian distribution with $\mathbf{q}(k) \sim \mathcal{N}(0,0.01)$, where $\mathcal{N}(0,0.01)$ denotes a zero-mean Gaussian distribution with variance 0.01 .

\subsubsection{Estimation results of different algorithms}

The following simulation results are presented to confirm

\begin{tabular}{|c|c|c|c|c|}
\hline Algorithms & \multicolumn{2}{|c|}{ MCKF } & \multicolumn{2}{|c|}{ MEE-KF } \\
\hline Parameters & $\sigma$ & $\varepsilon$ & $\sigma$ & $\varepsilon$ \\
\hline Gaussian noise & 10 & $15^{-6}$ & 10 & $10^{-6}$ \\
\hline Gaussian noise with outliers & 6.0 & $10^{-6}$ & 2.0 & $10^{-6}$ \\
\hline mixture Gaussian noise & 6.0 & $10^{-6}$ & 2.0 & $10^{-6}$ \\
\hline $\begin{array}{l}\text { mixture Gaussian noise with } \\
\text { outliers }\end{array}$ & 5.0 & $10^{-6}$ & 1.5 & $10^{-6}$ \\
\hline
\end{tabular}
the desirable performance of the MEE-KF. Here, we use 30000 samples (time steps) to calculate MSE.
Table 3

Parameter Settings of MCKF and MEE-KF

First, the influence of the measurement noises on MSE is investigated. For the distribution of $\mathbf{r}(k)$, we consider four cases: (1) Gaussian noise: $\mathbf{r}(k) \sim$ $\mathcal{N}(0,0.05)$; (2) Gaussian noise with outliers: $\mathbf{r}(k) \sim$ $0.99 \mathcal{N}(0,0.009)+0.01 \mathcal{N}(0,1000)$, where $\mathcal{N}(0,1000)$ is used to generate outliers; (3) mixture Gaussian noise: $\mathbf{r}(k) \sim 0.01 \mathcal{N}(-0.1,0.001)+0.99 \mathcal{N}(0.1,1000)$; (4) mixture Gaussian noise with outliers: $\mathbf{r}(k) \sim$ $0.48 \mathcal{N}(-0.1,0.001)+0.04 \mathcal{N}(0,1000)+0.48 \mathcal{N}(0.1,0.001)$. Table 2 gives the estimation results of $\mathrm{KF}, \mathrm{MCKF}$ and $\mathrm{MEE}-\mathrm{KF}$ in the presence of different measurement noises. The parameter settings of MCKF and MEE-KF are given in Table 3, and all these parameters are set by trials to obtain the best performance. From Table 2, we observe: i) under the Gaussian noises, the performance of MCKF approaches that of KF, and KF preforms the optimal performance than MEE-KF; ii) when large outliers or mixture Gaussian noise (Case (2), Case (3) and Case (4)) are included in the measurement noise, the MCKF and MEE-KF show better performance than $\mathrm{KF}$, and the MEE-KF achieves the best performance among the three algorithms.

Next, we investigate the influence of kernel size on the estimation performance. Table 4 shows the MSE comparison of different filters with different kernel sizes, where 
Table 4

Estimation Results of Different Algorithms with Different Kernel Sizes

\begin{tabular}{llllll}
\hline Algorithms & $\sigma$ & MSE of x1 & MSE of x2 & MSE of x3 & MSE of x4 \\
\hline KF & N /A & $1.2031 \pm 0.3572$ & $1.1811 \pm 0.2139$ & $0.3901 \pm 0.6712$ & $0.3622 \pm 0.2057$ \\
& $\sigma=3.0$ & $N / A$ & $N / A$ & $N / A$ & $N / A$ \\
& $\sigma=4.0$ & $0.8213 \pm 0.5663$ & $0.8437 \pm 0.3573$ & $0.3773 \pm 0.7391$ & $0.3298 \pm 0.2330$ \\
& $\sigma=5.0$ & $0.8423 \pm 0.4875$ & $0.8121 \pm 0.2310$ & $0.3610 \pm 0.0495$ & $0.3260 \pm 0.0352$ \\
& $\sigma=10.0$ & $1.0576 \pm 0.3515$ & $1.0366 \pm 0.2103$ & $0.3766 \pm 0.6477$ & $0.3471 \pm 0.2066$ \\
MEE-KF & $\sigma=20$ & $1.1668 \pm 0.3709$ & $1.1748 \pm 0.2570$ & $0.3895 \pm 0.6414$ & $0.3615 \pm 0.2667$ \\
& $\sigma=1.0$ & $1.7206 \pm 0.3869$ & $0.5587 \pm 0.3001$ & $0.3593 \pm 0.5045$ & $0.2896 \pm 0.1772$ \\
& $\sigma=1.5$ & $\mathbf{0 . 6 2 5 8} \pm \mathbf{0 . 2 9 4 2}$ & $\mathbf{0 . 5 3 7 6} \pm \mathbf{0 . 4 9 4 3}$ & $\mathbf{0 . 3 2 9 6} \pm \mathbf{0 . 4 9 3 0}$ & $\mathbf{0 . 2 8 9 6} \pm \mathbf{0 . 2 4 4 1}$ \\
& $\sigma=2.0$ & $0.6833 \pm 0.3411$ & $0.6671 \pm 0.6711$ & $0.3490 \pm 0.4930$ & $0.4213 \pm 0.2453$ \\
& $\sigma=3.0$ & $0.8410 \pm 0.2367$ & $0.9269 \pm 0.2604$ & $0.3945 \pm 0.4748$ & $0.5876 \pm 0.3474$ \\
& $\sigma=5.0$ & $1.0381 \pm 0.2719$ & $1.1381 \pm 0.3910$ & $0.4391 \pm 0.4750$ & $0.7279 \pm 0.2161$ \\
& $\sigma=10$ & $1.1912 \pm 0.2995$ & $1.2142 \pm 0.3956$ & $0.4628 \pm 0.4698$ & $0.7814 \pm 0.2204$ \\
\hline
\end{tabular}

the measurement noise is the same as Case (4). The thresholds in MCKF and MEE-KF are set to $10^{-6}$. From Table 4, we find out that if the kernel size is too small or too large, the performance of MCKF and MEE-KF will become worse. Especially, for the small kernel size, the MCKF may diverge, while MEE-KF is more stable compared with MCKF. The desirable performance can be achieved by MEE-KF when the kernel size is around 1.5 .

Finally, the computing time of different filters at each iteration is summarized in Table 5, which is measured with MATLAB 2016b running on i5-4590 and 3.30 GHZ CPU. We see that the MEE-KF can achieve better performance at the cost of slightly higher computational burden than MCKF.

\subsection{Tracking of Autonomous Driving}

This experiment shows the tracking of autonomous driving with the Kalman fusion algorithms [42]. The data source is from Udacity course self-driving car [43], which is measured by the lidar and radar sensors. In general, different sensors provide different types of information with different accuracies, especially in different weather conditions [44]. In this example, the lidar sensor provides a good resolution about the position, while the radar sensor provides better accuracy about the velocity in poor weather compared to lidar. To merge the information from different sensors, the Kalman fusion algorithms are applied to different sensors to estimate effectively the trajectory of vehicle. Fig. 1 shows the Kalman fusion model, where $\mathbf{x}(k-1)$ denotes the state variables at time $k-1 ; \mathbf{y}_{L}(k)$ and $\mathbf{y}_{R}(k)$ are the observations from lidar and radar sensors; $\hat{\mathbf{x}}_{L}(k)$ and $\hat{\mathbf{x}}_{R}(k)$ are the estimated states; $\hat{\mathbf{x}}(k)$ is the fusion state at time $k$. The detailed descriptions are presented in the following.
Table 5

Average Computing Time (sec) for Each Iteration

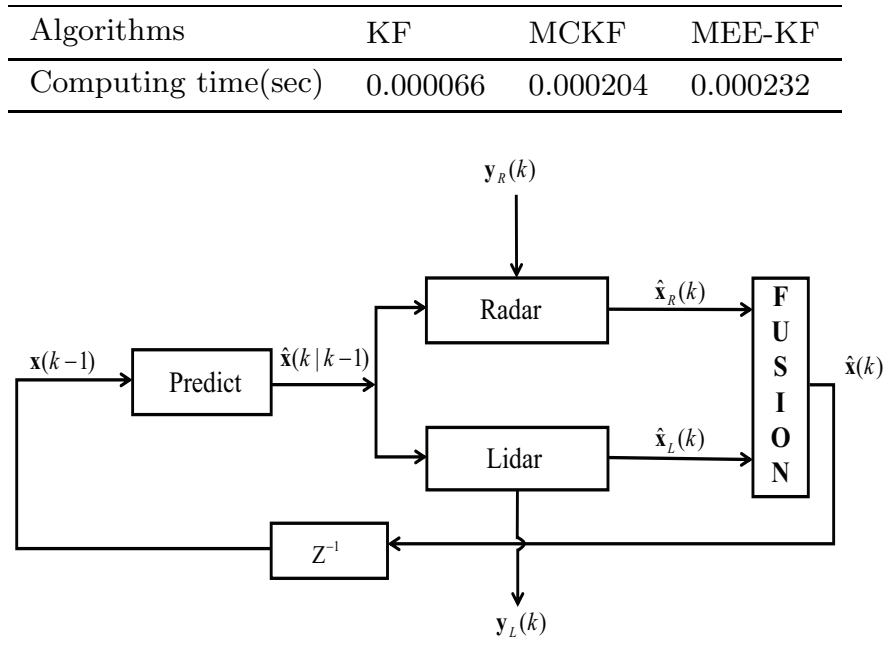

Fig. 1. Kalman fusion model.

\subsubsection{Model}

For the tracked vehicle, the state can be described by a 4 dimensional vector $\mathbf{x}(k)=\left[p_{x}(k), p_{y}(k), v_{x}(k), v_{y}(k)\right]^{\mathrm{T}}$, where $p_{x}(k)$ and $p_{y}(k)$ denote the position information of $x$ and $y$ coordinates; $v_{x}(k)$ and $v_{y}(k)$ are the corresponding velocity information. The state equation can be expressed by

$$
\mathbf{x}(k)=\left[\begin{array}{cccc}
1 & 0 & \Delta T & 0 \\
0 & 1 & 0 & \Delta T \\
0 & 0 & 1 & 0 \\
0 & 0 & 0 & 1
\end{array}\right] \mathbf{x}(k-1)+\mathbf{q}(k),
$$




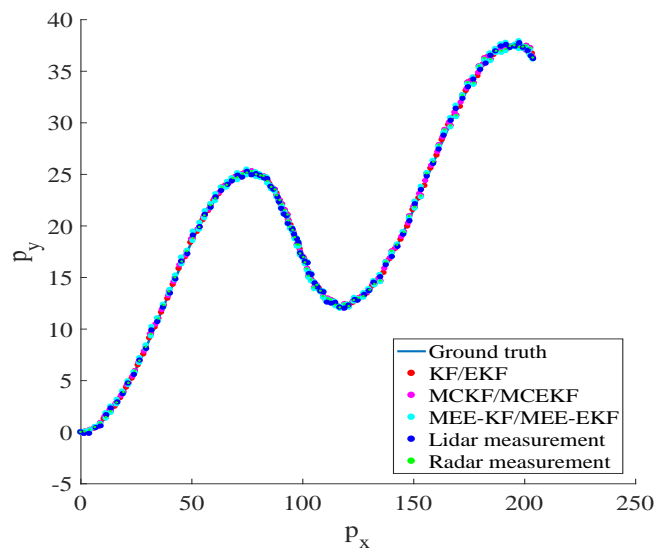

Fig. 2. Tracking results of different filters for trajectory 1 .

where $\Delta T=0.1$ sec is the time interval and $\mathbf{q}(k)$ is the process noise with covariance matrix $\mathbf{Q}(k)=$ $\left[\begin{array}{cccc}\frac{\Delta T^{2}}{4} & 0 & \frac{\Delta T^{3}}{2} & 0 \\ 0 & \frac{\Delta T^{2}}{4} & 0 & \frac{\Delta T^{3}}{2} \\ \frac{\Delta T^{3}}{2} & 0 & \Delta T^{2} & 0 \\ 0 & \frac{\Delta T^{3}}{2} & 0 & \Delta T^{2}\end{array}\right]$. The prior error covariance matrix is initialized as $\mathbf{P}(1 \mid 0)=\operatorname{diag}[1,1,1000,1000]$.

For the measurement equation, we consider two cases: Case I: When the measurements $\mathbf{y}_{L}(k)=\left[p_{x}(k), p_{y}(k)\right]^{\mathrm{T}}$ are acquired from the lidar sensor, the measurement equation can be described as

$$
\mathbf{y}_{L}(k)=\left[\begin{array}{llll}
1 & 0 & 0 & 0 \\
0 & 1 & 0 & 0
\end{array}\right] \mathbf{x}(k)+\mathbf{r}_{L}(k)
$$

where $\mathbf{r}_{L}(k)$ is the measurement noise from lidar sensor with covariance matrix $\mathbf{R}_{L}(k)=\operatorname{diag}[0.0025,0.0025]$. For the linear state space model in (89) and (90), the KF, MCKF and MEE-KF can be employed.

Case II: The measurements $\mathbf{y}_{R}(k)=[\rho(k), \varphi(k), \bar{\rho}(k)]^{\mathrm{T}}$ from the radar sensor involve three components in polar coordinates, i.e., the range $\rho(k)$, the angle $\varphi(k)$ between $\rho(k)$ and $x$ coordinates axis, and the range rate $\bar{\rho}(k)$. The measurement equation can then be established by:

$$
\mathbf{y}_{R}(k)=\mathbf{h}(\mathbf{x}(k))+\mathbf{r}_{R}(k),
$$

where the nonlinear function

$$
\mathbf{h}(\mathbf{x}(k))=\left[\begin{array}{c}
\sqrt{p_{x}^{2}(k)+p_{y}^{2}(k)} \\
\arctan \left(\frac{p_{y}(k)}{p_{x}(k)}\right) \\
\frac{p_{x}(k) v_{x}(k)+p_{y}(k) v_{y}(k)}{\sqrt{p_{x}^{2}(k)+p_{y}^{2}(k)}}
\end{array}\right]
$$

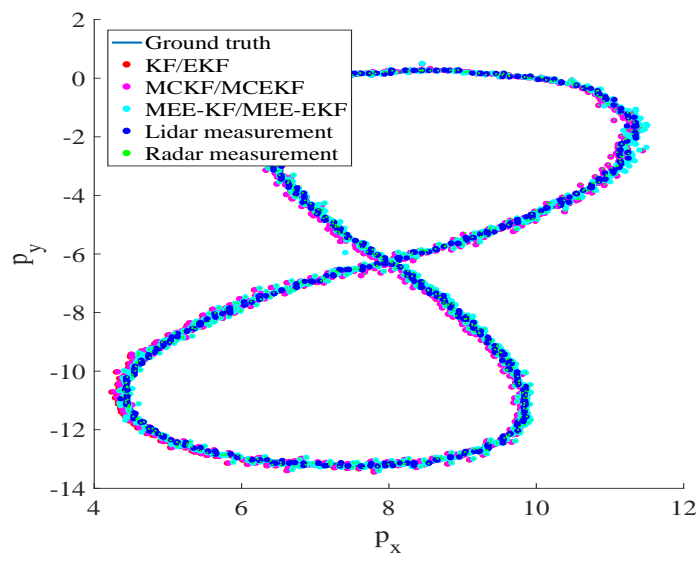

Fig. 3. Tracking results of different filters for trajectory 2 .

can be derived by a mapping from the polar coordinates to the cartesian coordinates; $\mathbf{r}_{R}(k)$ is the measurement noise from radar sensor with covariance matrix $\mathbf{R}_{R}(k)=\operatorname{diag}[0.09,0.05,0.09]$. To estimate the state $\mathbf{x}(k)$ in the cartesian coordinates, one should transform the measurements $\mathbf{y}_{R}(k)=[\rho(k), \varphi(k), \bar{\rho}(k)]^{\mathrm{T}}$ as state vector $\mathbf{x}(k)=\left[p_{x}(k), p_{y}(k), v_{x}(k), v_{y}(k)\right]^{\mathrm{T}}$ :

$$
\begin{array}{ll}
p_{x}(k)=\rho(k) \cos (\varphi(k)), & p_{y}(k)=\rho(k) \sin (\varphi(k)) \\
v_{x}(k)=\bar{\rho}(k) \cos (\varphi(k)), & v_{y}(k)=\bar{\rho}(k) \sin (\varphi(k)) .
\end{array}
$$

For the nonlinear state space model in (89) and (91), the EKF, MCEKF and MEE-EKF can be used.

\subsubsection{Estimation results of different algorithms}

The compared algorithms are performed for two trajectories, i.e., trajectory 1 and trajectory 2. Fig. 2 and Fig. 3 show the tracking results of trajectory 1 and trajectory 2 , respectively. Table 6 presents the MSE of different Kalman fusion algorithms, i.e., the KF/EKF, $\mathrm{MCKF} / \mathrm{MCEKF}$ and MEE-KF/MEE-EKF. In this simulation, the kernel sizes are set to 1.66 in MEE-EKF, 20.0 in MEE-KF, 15.0 in MCC-EKF and 20.0 in MCC-KF. From Table 6, one can see that the MEE-KF/MEE-EKF fusion method exhibits the best performance among all compared algorithms.

\subsection{Prediction of Infectious Disease Epidemics}

In this part, the prediction of epidemic [45] is used to validate the effectiveness of the proposed MEE-EKF. The disease control center (DCC) provides the InfluenzaLike-Illness (ILI) data [45] of the United States, a traditional information source for the epidemic estimation. 
Table 6

Estimation Results of Different Algorithms for Trajectory 1 and Trajectory 2

\begin{tabular}{llll}
\hline Algorithms & KF/EKF & MCKF/MCEKF & MEE-KF/MEE-EKF \\
\hline MSE of trajectory 1 & 0.5408 & 0.2570 & 0.1554 \\
MSE of trajectory 2 & 0.008887 & 0.008184 & 0.007789 \\
\hline
\end{tabular}

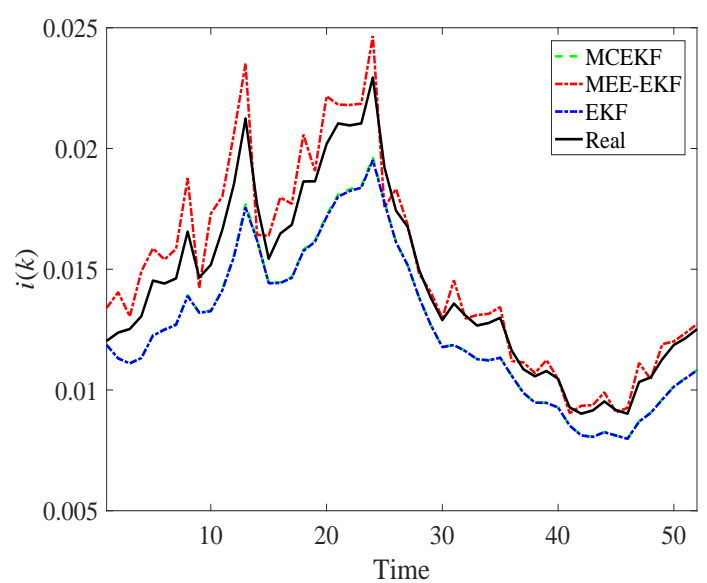

Fig. 4. Estimation results for infected $i(k)$ by EKF, MCEKF and MEE-EKF.

\subsubsection{Model}

The state process of an epidemic is based on the Susceptible-Infected-Recovered (SIR) model [45], given by

$$
\begin{aligned}
& s(k+1)=s(k)-\delta_{1} s(k) i(k) \\
& i(k+1)=i(k)+\delta_{1} s(k) i(k)-\delta_{2} i(k) \\
& b(k+1)=b(k)+\delta_{2} i(k),
\end{aligned}
$$

where $s(k), i(k)$ and $b(k)$ are the susceptible, infected and recovered crowd density, respectively, with $s(k)+$ $i(k)+b(k)=1$ for all $k$. The parameter $\delta_{1}$ denotes the rate about the spread of illness and $\delta_{2}$ is the rate about recovery from infection. Since the recovered $b(k)$ is obtained by $b(k)=1-s(k)-i(k)$, the state of epidemic at time $k$ can be described by $\mathbf{x}(k)=[s(k), i(k)]^{\mathrm{T}}$. Therefore, the state equation can be written by

$$
\mathbf{x}(k)=\mathbf{f}(\mathbf{x}(k-1))+\mathbf{q}(k),
$$

where the nonlinear function $\mathbf{f}(\mathbf{x}(k-1))$ is given in Eqs. (94) and (95), and $\mathbf{Q}(k)=10^{-8} \mathbf{I}_{n}$.

In the state space model, the measurement equation specifies how the observed data depend on the state of epidemic. When monitoring an epidemic, the unknown $s(k), i(k)$, and $b(k)$ are regarded as hidden states of the model, and the observed data are acquired via syndromic surveillance system. Due to only $i(k)$ can be obtained by the syndromic surveillance system, the measurement equation can be established by

$$
y(k)=\mathbf{H} \mathbf{x}(k)+r(k)
$$

Table 7

Performance Comparison of Different Algorithms for Prediction of Infectious Disease Epidemics

\begin{tabular}{llll}
\hline Algorithms & EKF & MCEKF & MEE-EKF \\
\hline MSE & 0.001729 & 0.001703 & 0.000825 \\
\hline
\end{tabular}

where the matrix $\mathbf{H}=\left[\begin{array}{ll}0 & 1\end{array}\right]$ denotes the measurement matrix and $\mathbf{R}(k)=10^{-6}$.

\subsubsection{Estimation results of different algorithms}

Figure 4 plots 52 weeks density of people infected in 2010 year. Table 7 gives the estimated MSE by EKF, MCEKF and MEE-EKF algorithms. In Fig. 4, a peak around the 25th week occurs, which means that the number people infected arrives at maximum. In the SIR model, the contact rate $\delta_{1}$ and recovery time $\delta_{2}$ are set to 0.35 and 0.11 , respectively. The kernel sizes of MEE-EKF and MCEKF are 20.0, and 16.0, respectively. From Fig. 4 and Table 7 , the MEE-EKF provides a much better prediction for the density of people infected than EKF and MCEKF.

\section{Conclusion}

In this paper, we propose the minimum error entropy Kalman filter (MEE-KF) with a fixed-point iteration. Unlike the original Kalman filter (KF) based on the wellknown minimum mean square error (MMSE) criterion and the maximum correntropy Kalman filter (MCKF) based on the maximum correntropy criterion (MCC), the MEE-KF is developed by using the minimum error entropy (MEE) criterion as the optimality criterion. With an appropriate kernel size, the MEE-KF algorithm can achieve better performance than the KF and MCKF especially when the underlying system is disturbed by some complicated non-Gaussian noises. The computational complexity of the MEE-KF is provided, and a sufficient condition for the convergence of the fixed-point iteration is given. Furthermore, the MEE based extended Kalman filter (MEE-EKF) is also developed to deal with the problem of state estimation of a nonlinear system in non-Gaussian noises. Simulations on land vehicle navigation, tracking of autonomous driving and prediction of infectious disease epidemics have confirmed the desirable performance of the proposed MEE-KF and MEEEKF. 


\section{Acknowledgements}

This work was supported by the 973 Program (No. 2015CB351703), National Key R\&D Program of China (No. 2017YFB1002501), and National NSF of China (No. 91648208, No. U1613219).

\section{References}

[1] R. E. Kalman, A new approach to linear filtering and prediction problem, Trans. ASME, Ser. D, J. Basic Eng., pages 5-45, 1960.

[2] G. Welch and G. Bishop, An Introduction to the Kalman Filter, Univ. North Carolina, Chapel Hill, NC, USA, Lecture, 2001.

[3] J. K. Uhlman, Algorithms for multiple target tracking, Amer. Sci., 80(2):128-141, 1997.

[4] B. Terzic and M. Jadric, Design and implementation of the extended kalman filter for the speed and rotor position estimation of brushless DC motor, IEEE Trans. Ind. Electron., 48(6):1065-1073, 2001.

[5] S. Y. Chen, Kalman filter for robot vision: a survey, IEEE Trans. Ind. Electron., 59(11):4409-4420, 2012.

[6] Y. Huang, Y. Zhang, Zhemin. Wu, N. Li, and J. Chambers, A novel adaptive Kalman filter with inaccurate process and measurement noise covariance matrices, IEEE Trans. Automat. Contr., 63(2):594-601, 2017.

[7] S. Julier and J. Uhlmann, A new extension of the Kalman filter to nonlinear systems, in Proc. 11th Int. Symp. Aerosp./Defence Sens., Simul. Contr., pages 182-193, 1997.

[8] Y. Huang, Y. Zhang, B. Xu, Z. Wu, and J. A. Chambers, A new adaptive extended Kalman filter for cooperative localization, IEEE Trans. Aerosp. Electron. Syst., 54(1):353368, 2018.

[9] E. A. Wan, R. V. Merwe, and A. T. Nelso, Dual estimation and the unscented transformation, in Adv. Neural Inf. Process. Syst., Cambridge, MA, USA: MIT Press, pages 666$672,2000$.

[10] I. Arasaratnam, S. Haykin, and T. R. Hurd, Cubature Kalman filtering for continuous-discrete systems: Theory and simulations, IEEE Trans. Signal Process., 58(6):49774993,2010.

[11] J. C. Príncipe, Information Theoretic Learning: Renyis Entropy and Kernel Perspectives; Springer: New York, NY, USA, 2010.

[12] B. Chen, Y. Zhu, J. Hu, J. C. Príncipe, System Parameter Identification: Information Criteria and Algorithms. USA, NY, New York: Newnes, 2013.

[13] W. Liu, P. Pokharel, J. C. Príncipe, Correntropy: properties and applications in non-Gaussian signal processing, IEEE Trans. Signal Process., 55(11):5286-5298, 2007.

[14] A. Singh, J. C. Príncipe, Using correntropy as a cost function in linear adaptive filters, in Proc. Int. Joint Conf. Neural Netw. (IJCNN), pages 2950-2955, 2009.

[15] B. Chen, X. Liu, H. Zhao, and J. C. Príncipe, Maximum correntropy Kalman filter, Automatica, 76:(70-77), 2017.

[16] G. T. Cinar and J. C. Príncipe, Hidden state estimation using the correntropy filter with fixed point update and adaptive kernel size, in Proc. Int. Joint Conf. Neural Netw. (IJCNN), pages 1-6, 2012.
[17] R. Izanloo, S. A. Fakoorian, H. S. Yazdi, and D. Simon, Kalman filtering based on the maximum correntropy criterion in the presence of non-Gaussian noise, in Annual Conf. Inf. Sci. Syst. (CISS), pages 500-505, 2016.

[18] M. V. Kulikova, Square-root algorithms for maximum correntropy estimation of linear discrete-time systems in presence of non-Gaussian noise. Syst. Control Lett., 108:8-15, 2017.

[19] M. V. Kulikova, Sequential maximum correntropy Kalman filtering, Asian J. Control, 21(6):1-9, 2019

[20] M. V. Kulikova, Factored-form Kalman-like implementations under maximum correntropy criterion, Signal Process., 160:328-338, 2019.

[21] Y. D. Wang, W. Zheng, S. M. Sun, and L. Li, Robust information filter based on maximum correntropy criterion, J. Guid., Control, Dyn., 39:1126-1131, 2016.

[22] B. Hou, Z. He, X. Zhou, H. Zhou, D. Li, and J. Wang, Maximum correntropy criterion Kalman filter for $\alpha$-Jerk tracking model with non-Gaussian noise, Entropy, 19(12):648, 2017.

[23] E. Makridis, K, M. Deliparaschos, E. Kalyvianaki, and T. Charalambous, Dynamic CPU resource provisioning in virtualized servers using maximum correntropy criterion Kalman filters, IEEE Int. Conf. Emerging Tech. Factory Automat. (ETFA), pages 12-15, 2018.

[24] G. Wang, R. Xue, and J. Wang, A distributed maximum correntropy Kalman filter, Signal Process., 160:241-247, 2019.

[25] Xi. L, Q. Hua, J. Zhao, and B. Chen, Extended Kalman filter under maximum correntropy criterion, in Proc. Int. Joint Conf. Neural Netw. (IJCNN), pages 1733-1737, 2016.

[26] F. Ma, F. Liu, X. Zhang, and P. Wang, An ultrasonic positioning algorithm based on maximum correntropy criterion extended Kalman filter weighted centroid, Signal, Image Video Process., 12(6):1207-1215, 2018.

[27] S. Mohiuddin and J. Qi, Maximum correntropy extended Kalman filtering for power system dynamic state estimation, IEEE Power Energy Soc. General Meeting, 2019.

[28] X. Liu, B. Chen, B. Xu, Z. Wu, and P. Honeine, Maximum correntropy unscented filter, Int. J. Syst. Sci, 48(8):1607$1615,2017$.

[29] G. Wang, N. Li, and Y. Zhang, Maximum correntropy unscented Kalman and information filters for non-Gaussian measurement noise, J. Frank. Inst., 354(18):8659-8677, 2017.

[30] B. Hou, Z. He, D. Li, H. Zhou, and J. Wang, Maximum correntropy unscented Kalman filter for ballistic missile navigation system based on SINS/CNS deeply integrated mode, Sensors, 18(6):1724, 2018.

[31] X. Liu, H. Qu, J. Zhao, and P. Yue, Maximum correntropy square-root cubature Kalman filter with application to SINS/GPS integrated systems, ISA Trans., 8(9):195-202, 2018.

[32] W. Qin, X. Wang, and N. Cui, Maximum correntropy sparse Gauss-Hermite quadrature filter and its application in tracking ballistic missile, IET Radar Sonar Navigat., 11(9):1388-1396, 2017.

[33] B. Chen, J. C. Príncipe. Maximum correntropy estimation is a smoothed MAP estimation, IEEE Signal Process. Lett. 19(8):491-494, 2012.

[34] D. Erdogmus, J. C. Príncipe. An error-entropy minimization for supervised training of nonlinear adaptive systems, IEEE Trans. Signal Process., 50(7):1780-1786, 2002. 
[35] Y. Zhang, B. Chen, X. Liu, Z. Yuan, and J. C. Príncipe, Convergence of a fixed-point minimum error entropy algorithm, Entropy, 17(8):5549, 2015.

[36] D. Erdogmus, J. C. Príncipe, Generalized information potential criterion for adaptive system training, IEEE Trans. Neural Netw., 13(5):1035-1044, 2002.

[37] L. M. Silva, C. S. Felgueiras, L. A. Alexandre, and de S J. Marques, Error entropy in classification problems: A univariate data analysis, Neural computat., 18(9):2036-2061, 2006.

[38] I. Santamaria, D. Erdogmus, and J. C. Príncipe. Entropy minimization for supervised digital communications channel equalization, IEEE Trans. Signal Process., 50(5):1184-1192, 2002.

[39] S. Peng, W. Ser, B. Chen, L. Sun, and Z. Lin, Robust constrained adaptive filtering under minimum error entropy criterion, IEEE Trans. Circuits Syst. II: Exp. Briefs, 65(8):1119-1126, 2018.

[40] B. Chen, Z. Yuan, N. Zheng, and J. C. Príncipe, Kernel minimum error entropy algorithm, Neurocomput., 121:160169, 2013.

[41] B. Chen, L. Xing, B. Xu, H. Zhao, and J. C. Príncipe. Insights into the robustness of minimum error entropy estimation, IEEE Trans. Neural Netw. Learn. Syst., 29(3):731-737, 2018.

[42] C. Blanc, L. Trassoudaine, and J. Gallice, EKF and particle filter track-to-track fusion: A quantitative comparison from radar/lidar obstacle tracks, in Proc. 8th Int. Conf. Inf. Fusion, pages 1303-1310, 2005.

[43] Udacity, Udacitys Self-Driving Car Simulator, https:// github.com/udacity/self-driving-car-sim.

[44] O. Cameron, Race Self-Driving Cars With Udacity-Udacity Inc-Medium, https://medium.com/udacity/raceself-drivingcars-with-udacity-42ae12e545c1.

[45] E. Ray, N. Reich, Prediction of infectious disease epidemics via weighted density ensembles, PLoS computat. biol., 14(2), 2018. 